$$
\begin{array}{r}
\text { Pontifícia Universidade Católica } \\
\text { do Rio de Janeiro }
\end{array}
$$

Fernanda Magalhães Rumenos Guardado

\title{
Estudo sobre a IS Intertemporal na Economia Brasileira
}

\section{Dissertação de Mestrado}

Dissertação apresentada como requisito parcial para obtenção do título de Mestre pelo Programa de Pós-Graduação em Economia da PUC-Rio.

Orientadores: Eduardo Henrique M. Motta Loyo Marcelo Cunha Medeiros 


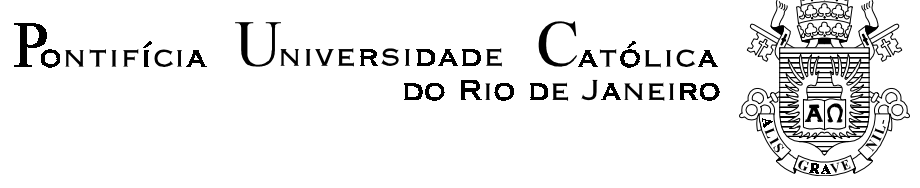

Fernanda Magalhães Rumenos Guardado

\section{Estudo sobre a IS Intertemporal na Economia Brasileira}

Dissertação apresentada como requisito parcial para obtenção do título de Mestre pelo Programa de PósGraduação em Economia da PUC-Rio. Aprovada pela Comissão Examinadora abaixo assinada.

Eduardo Henrique M. Motta Loyo

Orientador

Departamento de Economia - PUC-Rio

Marcelo Cunha Medeiros

Co-Orientador

Departamento de Economia - PUC-Rio

Márcio Gomes Pinto Garcia

Departamento de Economia - PUC-Rio

João Pontes Nogueira

Vice-Decano de Pós-Graduação do CCS - PUC-Rio

Rio de Janeiro, 12 de abril de 2004 
Todos os direitos reservados. É proibida a reprodução total ou parcial do trabalho sem autorização da universidade, da autora e do orientador.

\section{Fernanda Magalhães Rumenos Guardado}

Graduou-se em Ciências Econômicas na PUC-Rio em 2001. Cursou o mestrado de Economia da PUC-Rio entre 2002 e 2003. Interesse acadêmico na pesquisa relativa à política monetária e modelos estruturais.

Ficha Catalográfica

Guardado, Fernanda Magalhães Rumenos

Estudo sobre a IS intertemporal na economia brasileira / Fernanda Magalhães Rumenos Guardado; orientadores: Eduardo Henrique M. Motta Loyo, Marcelo Cunha Medeiros - Rio de Janeiro : PUC, Departamento de Economia, 2004.

64 f. : il. ; $30 \mathrm{~cm}$

Dissertação (mestrado) - Pontifícia Universidade Católica do Rio de Janeiro, Departamento de Economia.

Inclui referências bibliográficas.

1. Economia - Teses. 2. IS intertemporal. 3. Modelos estruturais. 4. Curva de demanda. 5. Formação de hábito. 6. Equação de Euler. I. Loyo, Eduardo Henrique M. Motta. II. Medeiros, Marcelo Cunha. III. Pontifícia Universidade Católica do Rio de Janeiro. Departamento de Economia. IV. Título. 
Para meus pais, Lincoln e Marli, cujo apoio foi fundamental para o término e sucesso de mais esta etapa; a Paulo, cuja paciência e compreensão tornou todo este período menos sacrificante e penoso. 


\section{Agradecimentos}

Aos meus orientadores, pelo estímulo e por terem me ensinado tanto sobre pesquisa no decorrer deste trabalho.

Aos meus pais, pelo carinho, apoio e compreensão que só vocês poderiam ter.

Ao meu namorado, Paulo, pela paciência e compreensão.

Aos meus irmãos, Soraya e Daniel.

Ao CNPq, pelo auxílio concedido, sem o qual este trabalho não poderia ter sido realizado.

Aos professores que participaram da Comissão examinadora.

Aos meus colegas da PUC. 


\section{Resumo}

Guardado, Fernanda Magalhães R. . Estudo sobre a IS Intertemporal na Economia Brasileira. Rio de Janeiro, 2004. 64p. Dissertação de Mestrado - Departamento de Economia, Pontifícia Universidade Católica do Rio de Janeiro.

A IS intertemporal, que representa a dinâmica da Demanda Agregada em modelos estruturais que visem avaliar a política monetária, pode ter diferentes formatos dependendo das hipóteses que são feitas a respeito da estrutura da economia. Neste trabalho buscou-se modelar as diferentes hipóteses, tais como formação de hábito de um e dois períodos, de maneira independente da política monetária e testar seu ajuste aos dados. Os resultados indicam que não só é importante introduzir defasagens do hiato do produto na regressão (tanto para aumentar seu poder de explicação quanto para retirar a autocorrelação dos resíduos), como que a taxa de juros só consegue ter coeficiente significantemente diferente de zero se for incluída na regressão a curva de juros nominais futuros. Entretanto, tais resultados são viesados pela amostra escolhida, um período que apresentou uma série de taxa de juros com indícios de nãoestacionariedade.

\section{Palavras-chave}

IS Intertemporal; Curva de Demanda; modelos estruturais; Formação de Hábito; Demanda Agregada 


\section{Abstract}

Guardado, Fernanda Magalhães Rumenos. Estimating the Intertemporal IS Equation in the Brazilian Economy. Rio de Janeiro, 2004. 64p. MSc. Dissertation - Departamento de Economia, Pontifícia Universidade Católica do Rio de Janeiro.

The intertemporal IS equation, which replicate de dynamics of Agregate Demand in structural models that aim to evaluate monetary policy, might take different shapes depending on the assumptions made on the structure of the economy underlying it. In the present work were modeled different hypothesis about the economy, such as habit formation of one and two periods, independent of monetary policy and tested the fit of such equations to the observed data. The results indicate that not only it is important to introduce lags of the output gap in the regression (in order to both elevate its explaining power and to retrieve any autocorrelation of the residuals), and that interest rates can only have a coefficient significantly different from zero if an nominal yield curve is also included. But these results are biased by the time sample used, in which the interest rate were repeatedly raised, and therefore the series suggest some signs of non-stationarity, which may have had some effect in the results.

\section{Keywords}

Intertemporal IS; Demand Curve; structural models; Habit Formation; Agregate Demand 


\section{Sumário}

1 Introdução 10

2 O Modelo Teórico 12

2.1. O Modelo Básico 12

2.2. Formação de Hábito versus Bens Duráveis 15

2.3. As Equações Relevantes 18

2.3.1. Equações com dependência intertemporal 18

2.3.2. Equações Correspondentes 19

2.4. O Problema das Expectativas 22

2.5. Extensões 24

3 Séries e Estimação 29

3.1. Séries 29

3.2. Método de Previsão e Formação de Expectativas 32

3.2.1. Inflação Zero 34

3.2.2. Introdução da Constante 43

3.3. Verificação dos Resultados 49

3.3.1. Bootstrap 49

3.3.2. Validação Cruzada 50

4 Conclusão $\quad 52$

5 Referências Bibliográficas $\quad 55$

6 Apêndice $\quad 57$

6.1. Equação I

6.2. Equação II 59

6.3. Equação com $\beta$ variante no tempo 63

$\begin{array}{ll}7 \text { Anexo } & 65\end{array}$ 


\section{Lista de tabelas}

Figura 1 - IPCA, Taxa Selic e Índice de Produção Industrial 24

Tabela 1 - Principais estatísticas das séries 31

Figura 2 - Hiato do produto, Expectativas do IPCA (Focus) e Taxa Selic 31

Figura 3 - Série resultante da extração de tendência linear da Selic 32

Figura 4 - Previsões HW e Focus 34

Tabela 2 - Equação I 39

Tabela 4 - Equações com Selic sem tendência 41

Tabela 5 - Equações com EMBI+ Brasil 42

Tabela 6 - Equações I e II $\quad 47$

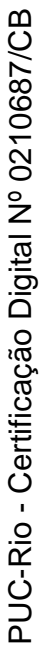

Tabela 7 - Equações I e II sem expectativas de hiato 48 